\title{
Embarrassment and behavioural changes among patients living with scars from cancer surgery: The appearance- related side effects and psychological distress
}

\author{
Mazzotti E ${ }^{1 *}$, Sebastiani $C^{2}$ and Marchetti $\mathbf{P}^{1,2}$ \\ ${ }^{1}$ Department of Molecular and Clinical Medicine, "Sapienza” University of Rome, Sant'Andrea Hospital, Rome, Italy \\ ${ }^{2}$ Fondazione per la Medicina Personalizzata, FMP, Rome, Italy
}

\begin{abstract}
Purpose: Cancer-related changes in appearance can be associated with a negative psychological status. This study examined the characteristics of embarrassment of scars due to cancer surgery and its relationship to psychological distress among patients with breast cancer and colorectal cancer.

Methods: Self-reported questionnaires were administered to 135 consecutive patients.

Results: $39.09 \%$ reported embarrassment in getting undressed in front of their partner and $64.42 \%$ at the gym. $49.49 \%$ reported changes in intimacy. $62.69 \%$ felt embarrassment and $64.18 \%$ reported discomfort due to their scars. $53.26 \%$ experienced distress due to hair loss and $44.83 \%$ due to temporary stomas. $19.31 \%$ viewed their appearance as defective and socially unacceptable, $25.03 \%$ assigned importance to physical attractiveness and unattractiveness, and $40.77 \%$ considered their appearance central to their identity and self-esteem. 77.04\% suffered psychological distress. Among patients with breast cancer, psychological distress was strongly associated with behavioral changes due to scars, self-care behaviors, low self-investment and lumpectomy. Among colorectal cancer patients, psychological distress was associated with colostomy.

Conclusions: Cancer-related scars assume importance at different levels and have different meanings among cancer patients: direct and physical versus indirect and relational. Our results showed that among colorectal cancer patients, the psychological distress was associated with stomas. On the other hand, our study found that among women with breast cancer psychological distress was associated with changes in the relationships with partners, others and with themselves.
\end{abstract}

\section{Introduction}

The destructive influence of scars and disfigurement on appearance and body image has been described [1-3]. Moreover, disturbances in body image are known to affect psychological adjustment in patients with cancer and many other chronic illnesses [4-9].

Among the cancer-related changes in appearance, scars can lead patients to considerable physical, psychological and relational changes. Different surgical procedures lead to relatively similar results in terms of quality of life, however patients that have a mastectomy, compared to those that have a breast-conserving surgery, usually report lower body image satisfaction and sexual functioning [10].

As clinicians well know, breast cancer patients frequently report embarrassment of their scars, saying that they cannot stand to look in the mirror nor to get undressed in front of others. Surgical treatment may cause disfigurement with varying degrees of observability, permanence, controllability and extensiveness. Some changes are easily observable to other people, such as loss of hair, colostomy and skin decolouration. Other changes are observable only to people close to the patient such as a partner, others are not observable, while some are temporary. Nevertheless secondary changes in psychological variables may not return to pre-surgery status [3]. It seems reasonable to hypothesize that having scars may result in significant daily stress that is highly subjective and does not necessary reflect objective reality.
The aims of this study were (1) to measure embarrassment and discomfort due to scars related to cancer surgery in patients with breast or colorectal cancer, (2) to measure the features of change and their relationships to psychological distress.

\section{Materials and methods}

One hundred thirty-five consecutive outpatients with cancer were enrolled in the study. The inclusion criteria were: cancer surgery to treat breast cancer or colorectal cancer; at least 18 years of age; absence of severe mental illness; written informed consent. The exclusion criteria were: patients enrolled in other studies, males with breast cancer.

Patients completed the study questionnaires before their appointment with a doctor and subsequently gave written informed consent. The study was approved by the Institutional Ethical Committee.

${ }^{\star}$ Correspondence to: Eva Mazzotti, Department of Molecular and Clinical Medicine, Sapienza University of Rome, Sant' Andrea Hospital, Rome, Italy, Tel: 39-06-33775831, E-mail: eva.mazzotti@gmail.com

Key words: breast cancer, colorectal cancer, embarrassment, investment in one's appearance, psychological distress, scars

Received: April 29, 2019; Accepted: May 27, 2019; Published: June 07, 2019 
Mazzotti E (2019) Embarrassment and behavioural changes among patients living with scars from cancer surgery: The appearance-related side effects and psychological distress

\section{Hospital anxiety depression scale (HADS)}

[11] The HADS is a 14-item questionnaire, (7 items for anxiety and 7 for depression) largely used as measure of psychological distress. Each item is rated on a four-point Likert-style format from 0 (not present) to 3 (highly present), and the total score thus ranges from 0 to 42 . A categorical measure of psychological distress (no cases/cases) was based on the cut-off point of 10/11 derived from the validation studies. The HADS internal consistency (Cronbach's $\alpha$ ) in this study was 0.88 .

\section{Appearance schemas inventory (ASI)}

[12] The 14 items of the ASI cover three domains: body-image vulnerability (BIV, 6 items), that involves a predisposition to view one's appearance as inherently, perhaps irreversibly defective and socially unacceptable; self-investment (SI, 5 items), that concerns beliefs that one's appearance is life-shaping and central to one's sense of self and that one must pursue and maximize physical attractiveness; and appearance stereotyping (AS, 3 items), that entails assumptions about the social meanings of physical attractiveness and unattractiveness. Exemplary items include: "I should do whatever I can to always look my best" and "My appearance is responsible for much of what has happened to me in my life". Higher scores reflect more psychological investment in one's appearance. The ASI's internal consistency (Cronbach's $\alpha$ ) in this study was 0.85 (BIV, $\alpha=0.72$; SI, $\alpha=0.74$; AS, $\alpha=0.67$ ).

Quality of life (qol), patient's perception of cancer severity and cancer curability

[13] were self-assessed by the patients using a 10-point rating scale from bad/very severe/very difficult to good/not severe/very easy.

For this study, we have used

Burden of scars was assessed by 2 items (How much are your scars a problem for you? How much do you feel embarrassed by your scars? Each item is rated on a 10-point scale, from "no/nothing" to "maximum problem").

Changes due to scars was assessed by 3 items (How much embarrassment do you feel when you get undressed in front of your partner?; How much embarrassment do you feel when you get undressed in front of others at the gym?; Have you experienced changes in intimacy due your scars?. These items are rated as "no/yes/ not applicable". The choice "not applicable" responds to: does not have partner; did not go to the gym; does not have sexual intimacy). A total score of Change has been obtained by adding the 3 items. The internal consistency (Cronbach's $\alpha$ ) was 0.80 .

Information about demographic, clinical and lifestyle variables (physical activity, intake of vitamins, meditation, psychotherapy, etc) were also collected. Clinical data (as data of diagnosis, metastasis, cancer-related symptoms) were extracted from hospital charts.

\section{Statistical analyses}

All scale scores were standardized on a 0 - to 100-point scale ( $\mathrm{T}$ values) and they have been calculated to allow comparability of scores across domains. Standard $\mathrm{T}$ scores are characterized by a distribution with a mean of 50 and a standard deviation of 10 (minimum $=0$, maximum $=100)$. ASI-subscale scores above the mean ( $\mathrm{T}$ score $>50)$ were considered indicators of dysfunctional schematic investment in appearance. Cases of psychological distress were operationally defined as patients scoring $>11$ on HADS. A measure of cancer-related symptoms (labelled Symptoms, ranging from 0 to 12) was obtained by making a total of the presence of each of 12 possible symptoms reported in the hospital chart (the list is reported in Table 2). A variable, labelled Self-care behavior has been obtained as the sum of non-smoker, non-drinker, presence of fruits and vegetables in the diet, practice of physical activities, use of massages, acupuncture, yoga, autogenic training, osteopathy, herbal medicine, and psychotherapy (theoretical range $0-12$ ).

For the purpose of analysis, we divided patients into two categories according to differences in education $(<=8$ years of school vs $>8 \mathrm{yrs})$, symptoms ( 0 vs $1+$ ), self-care behavior ( 0 vs $1+$ ), BMI ( $<25.0$ vs $=>25.0$ $\mathrm{kg} / \mathrm{m} 2$ ), self-perception of qol, disease severity and disease curability $(<=5$ vs $>5)$, and into 3 categories according to time since diagnosis $(<=12 ; 13-24 ; 24+)$.

Data are expressed as means and 95\% confidence intervals (CIs), or standard deviations (SDs), for continuous variables; as proportions for categorical variables. Differences between groups were tested using the Chi-squared or Fisher exact test, or t test as appropriate. Internal consistencies were calculated using Cronbach's a coefficients. To measure bivariate relationships the Pearson (r) coefficient was used.

Multivariate logistic regression models were built to assess the association between cases of psychological distress and all the variables resulted associated at the univariate analyses, while simultaneously adjusting for possible confounding factors, such as gender (only for patients with colorectal cancer), age, education and marital status. Only the variables significantly associated with the outcome of interest at a $\mathrm{p}<0.10$ level were retained in the final model.

Statistical analysis was performed using STATA, version 11.0 (StataCorp, College Station, Tex).

\section{Results}

Sixty-four women breast cancer patients aged from 24 to 83 years, and 71 colorectal cancer patients (43 males and 28 females) aged from 39 to 85 years, were enrolled in the study; their mean age was 53.6 years $(\mathrm{SD}=11.4)$ and $64,4(\mathrm{SD}=10.4)$, respectively $(\mathrm{p}<0.001)$ (Table 1$)$.

Among colorectal cancer patients, no differences were found between males and females with respect to age $(p=0.940)$, years of education $(p=0.683)$, first diagnosis of cancer $(p=0.689)$, time since diagnosis $(\mathrm{p}=0.855)$, metastasis at diagnosis $(\mathrm{p}=0.671)$, post-surgery chemotherapy $(\mathrm{p}=0.979)$, and radiotherapy $(\mathrm{p}=0.175)$. Differences emerged around marital status $(\mathrm{p}=0.053)$, females resulted more often divorced, separated or widowed, and about BMI ( $\mathrm{p}=0.092)$, males had more frequently a $\mathrm{BMI}>=25$.

Comparing patients with breast cancer and colorectal cancer we found differences in years of education $(p=0.002)$, the breast cancer patients had more frequently more than 8 years of education; marital status $(\mathrm{p}=0.050)$, but in this case the difference covered up gender difference; metastasis at diagnosis $(\mathrm{p}=0.004)$, more frequent in colorectal cancer patients; pre-surgery chemotherapy $(\mathrm{p}=0.002)$ and radiotherapy $(p<0.001)$, more frequently reported by breast cancer patients. No differences emerged for the other variables. In both samples about $70 \%$ of patients was at their first diagnosis of cancer; and close to $70 \%$ had a diagnosis in the previous 12 months; more than $90 \%$ had postsurgery chemotherapy. Among breast cancer patients, 33 (51.56\%) had a mastectomy, and 4 of them (6.25\%) a bilateral one. Among colorectal patients, $28(39.40 \%)$ had a stoma at the time of the study.

Cancer-related symptoms in patients with breast or colorectal cancer are reported in Table 2. Fatigue was the symptom more 
Mazzotti E (2019) Embarrassment and behavioural changes among patients living with scars from cancer surgery: The appearance-related side effects and psychological distress

Table 1. Sociodemographic and clinical characteristics according to types of cancer and gender

\begin{tabular}{|c|c|c|c|c|c|c|}
\hline & \multicolumn{2}{|c|}{ Colorectal } & & \multirow{2}{*}{$\begin{array}{c}\text { Colorectal }(\mathrm{N}=71) \\
\text { males+females }\end{array}$} & \multirow{2}{*}{$\begin{array}{c}\text { Breast }(N=64) \\
\text { females }\end{array}$} & \multirow[b]{3}{*}{ p-value } \\
\hline & males $N=43$ & females $N=\mathbf{2 8}$ & & & & \\
\hline & No.(\%)* & No.(\%)* & p-value & No. $(\%)^{*}$ & No.(\%)* & \\
\hline \multicolumn{7}{|l|}{ Age (years) } \\
\hline mean $(95 \% \mathrm{CI})$ & $64.44(61.28-67.60)$ & $64.30(60.10-68.40)$ & 0.940 & $64.37(61.91-66.82)$ & $53.58(50.72-56.44)$ & $<0.001$ \\
\hline \multicolumn{7}{|l|}{ Education (years) } \\
\hline$<=8$ & $24(55.81)$ & $17(60.71)$ & & $41(57.75)$ & $20(31.25)$ & \\
\hline$>8$ & $19(44.19)$ & $11(39.29)$ & 0.683 & $30(42.25)$ & $44(68.75)$ & 0.002 \\
\hline \multicolumn{7}{|l|}{ Marital status } \\
\hline married/cohabiting & $39(90.70)$ & $20(71.43)$ & & $59(83.10)$ & $42(65.63)$ & \\
\hline never married & $3(6.98)$ & $3(10.71)$ & & $6(8.45)$ & $8(12.50)$ & \\
\hline $\begin{array}{l}\text { divorced/separated/ } \\
\text { widowed }\end{array}$ & $1(2.33)$ & $5(17.86)$ & 0.053 & $6(8.45)$ & $14(21.88)$ & 0.050 \\
\hline \multicolumn{7}{|l|}{ BMI } \\
\hline$<25$ & $16(37.21)$ & $16(57.14)$ & & $32(45.07)$ & $34(53.13)$ & \\
\hline$>=25$ & $27(62.79)$ & $11(39.29)$ & 0.092 & $38(53.52)$ & $29(45.31)$ & 0.635 \\
\hline \multicolumn{7}{|l|}{$\begin{array}{c}\text { First diagnosis of } \\
\text { cancer }\end{array}$} \\
\hline no & $11(25.58)$ & $6(21.43)$ & & $17(23.94)$ & $20(31.25)$ & \\
\hline yes & $32(74.42)$ & $22(78.57)$ & 0.689 & $54(76.06)$ & $44(68.75)$ & 0.342 \\
\hline \multicolumn{7}{|l|}{$\begin{array}{c}\text { Time since diagnosis } \\
\text { (months) }\end{array}$} \\
\hline$<12$ & $30(69.77)$ & $19(67.86)$ & & $49(69.01)$ & $43(67.19)$ & \\
\hline $13-24$ & $3(6.98)$ & $3(10.71)$ & & $6(8.45)$ & $6(9.38)$ & \\
\hline$>24$ & $10(23.26)$ & $6(21.43)$ & 0.855 & $16(22.54)$ & $15(23.44)$ & 0.970 \\
\hline \multicolumn{7}{|l|}{ Metastasis at diagnosis } \\
\hline no & $18(41.86)$ & $13(46.43)$ & & $31(43.66)$ & $46(71.88)$ & \\
\hline yes & $21(48.84)$ & $11(39.29)$ & 0.671 & $32(45.07)$ & $14(21.88)$ & 0.004 \\
\hline \multicolumn{7}{|l|}{ Type of surgery } \\
\hline lumpectomia & & & & & $31(48.44)$ & \\
\hline mastectomy & & & & & $29(45.31)$ & \\
\hline mastectomy bilateral & & & & & $4(6.25)$ & \\
\hline stomia & $18(41.86)$ & $10(35.71)$ & & $28(39.4)$ & & \\
\hline \multicolumn{7}{|l|}{$\begin{array}{c}\text { Chemotherapy/pre- } \\
\text { surgery }\end{array}$} \\
\hline no & $40(93.02)$ & $28(100.0)$ & & $68(95.77)$ & $50(78.13)$ & \\
\hline yes & $3(6.98)$ & $0(0.00)$ & 0.153 & $3(4.23)$ & $14(21.88)$ & 0.002 \\
\hline \multicolumn{7}{|l|}{$\begin{array}{l}\text { Chemotherapy/post- } \\
\text { surgery }\end{array}$} \\
\hline no & $3(6.98)$ & $2(7.14)$ & & $5(7.04)$ & $2(3.13$ & \\
\hline yes & $40(93.02)$ & $26(92.86)$ & 0.979 & $66(92.96)$ & $62(96.88)$ & 0.305 \\
\hline \multicolumn{7}{|l|}{ Radiotherapy } \\
\hline no & $35(81.40)$ & $26(92.86)$ & & $61(85.92)$ & $38(59.38)$ & \\
\hline yes & $8(18.60)$ & $2(7.14)$ & 0.175 & $10(14.08)$ & $26(40.63)$ & $<0.001$ \\
\hline
\end{tabular}

*totals may vary because of missing value

frequently reported (61.48\%), followed by nausea (49.63\%), insomnia (38.52\%), and anxiety/stress (37.78\%). Nausea and anxiety/stress were more common among women; pain, fatigue, and muscular tension were more common in patients with breast cancer. Paresthesia and lymphedema were cancer-related specific symptoms. Vomiting $(\mathrm{N}=6$; $4.44 \%)$, cough $(\mathrm{N}=1 ; 0.74)$, and headache $(\mathrm{N}=1 ; 0.74)$ were sporadic symptoms (they were summed with all the other to obtain the variable "Symptoms"). Differences in Symptoms between colorectal cancer males and females (mean 2.12; 95\%CI 1.56-2.67 versus 2.86; $95 \% \mathrm{CI}$ 2.25-3.46; $\mathrm{p}=0.079$ ) and between colorectal cancer patients and breast cancer patients (mean 2.41 ; 95\%CI $2.00-2.82$ versus 3.44 ; 95\%CI 2.99 3.88; $\mathrm{p}<0.001$ ) have been found.

Lifestyle and use of non-conventional medicine are reported in Table 3. More than $77 \%$ of patients were non- or ex-smokers, $80 \%$ were non-drinkers, more than $77 \%$ had a moderate to high consumption of fruit and vegetables in their diet, $70 \%$ took part regularly in some sporting activity. Regarding the use of non-conventional medicine, $23.70 \%$ used herbal medicine or natural remedies (e.g: valeriana), $11.11 \%$ massages, $7.41 \%$ psychotherapy, $6.67 \%$ autogenic training, $4.44 \%$ acupuncture, $4.44 \%$ osteopathy, $3.70 \%$ yoga. Differences in selfcare behavior resulted between breast and colorectal cancer patients (mean 4.04; 95\%CI 3.74-4.34 versus 4.94; 95\%CI 4.52-5.36; $\mathrm{p}<0.001$ ). Women with breast cancer had more frequent self-care behavior (60.94\%) compared with women with colorectal cancer (46.43\%).

Eighty-four patients (62.69\%) felt embarrassment and 86 (64.18\%) reported discomfort due to their scars. 43 patients (39.09\%) reported embarrassment in getting undressed in front of their partner, $67(64.42 \%)$ at the gym, $49(49.49 \%)$ reported changes during sexual intimacy, 49 (53.26\%) suffered due to their hair loss, and $13(44.83 \%)$ due to a temporary colostomy. Descriptive statistics 
Mazzotti E (2019) Embarrassment and behavioural changes among patients living with scars from cancer surgery: The appearance-related side effects and psychological distress

Table 2. Cancer-related symptoms in patients with breast cancer or colorectal cancer

\begin{tabular}{|c|c|c|c|c|c|c|}
\hline & \multicolumn{2}{|c|}{ Colorectal } & & \multirow{2}{*}{$\begin{array}{c}\text { Colorectal }(\mathrm{N}=71) \\
\text { males+females }\end{array}$} & \multirow{2}{*}{$\begin{array}{c}\text { Breast }(N=64) \\
\text { females }\end{array}$} & \multirow[b]{3}{*}{ p-value } \\
\hline & males $N=43$ & females $N=\mathbf{2 8}$ & & & & \\
\hline & No.(\%)* & No.(\%)* & p-value & No.(\%)* & No.(\%)* & \\
\hline \multicolumn{7}{|l|}{ Anorexia } \\
\hline no & $43(100.0)$ & $26(92.86)$ & & $69(97.18)$ & $64(100.0)$ & \\
\hline yes & $0(0.00)$ & $2(7.14)$ & 0.075 & $2(2.82)$ & $0(0.00)$ & 0.176 \\
\hline \multicolumn{7}{|l|}{ Pain } \\
\hline no & 34 (79.07) & $22(78.57)$ & & $56(78.87)$ & $34(53.13)$ & \\
\hline yes & $9(20.93)$ & $6(21.43)$ & 0.960 & $15(21.13)$ & $30(46.88)$ & 0.002 \\
\hline \multicolumn{7}{|l|}{ Nausea } \\
\hline no & $33(76.74)$ & $10(35.71)$ & & $43(60.56)$ & $25(39.06)$ & \\
\hline yes & $10(23.26)$ & $18(64.29)$ & 0.001 & $28(39.44)$ & $39(60.94)$ & 0.013 \\
\hline \multicolumn{7}{|l|}{ Anxiety/stress } \\
\hline no & $33 / 76.74)$ & $15(53.57)$ & & $48(67.61)$ & $36(56.25)$ & \\
\hline yes & $10(23.26)$ & $13(46.43)$ & 0.041 & $23(32.39)$ & $28(43.75)$ & 0.174 \\
\hline \multicolumn{7}{|l|}{ Fatigue } \\
\hline no & $22(51.16)$ & $11(39.21)$ & & $33(46.48)$ & $19(29.69)$ & \\
\hline yes & $21(48.84)$ & $17(60.71)$ & 0.327 & $38(53.52)$ & $45(70.31)$ & 0.045 \\
\hline \multicolumn{7}{|l|}{ Insomnia } \\
\hline no & $27(62.79)$ & $20(71.43)$ & & $47(66.20)$ & $36(56.25)$ & \\
\hline yes & $16(37.21)$ & $8(28.57)$ & 0.452 & $24(33.80)$ & $28(43.75)$ & 0.236 \\
\hline \multicolumn{7}{|l|}{ Muscular tension } \\
\hline no & $34(79.07)$ & $22(78.57)$ & & $56(78.87)$ & $32(50.00)$ & \\
\hline yes & $9(20.93)$ & $6(21.43)$ & 0.960 & $15(21.13)$ & $32(50.00)$ & $<0.001$ \\
\hline \multicolumn{7}{|l|}{ Parestesie } \\
\hline no & $29(67.44)$ & $19(67.86)$ & & $48(67.61)$ & $64(100.0)$ & \\
\hline yes & $14(32.56)$ & $9(32.14)$ & 0.971 & $23(32.39)$ & $0(0.00)$ & $<0.001$ \\
\hline \multicolumn{7}{|l|}{ Lymphoedema } \\
\hline no & $43(100.0)$ & $28(100)$ & & $71(100.0)$ & $51(79.69)$ & \\
\hline yes & $0(0.00)$ & $0(0.00)$ & 1.000 & $0(0.00)$ & $13(20.31)$ & $<0.001$ \\
\hline \multicolumn{7}{|l|}{$\begin{array}{c}\text { Symptoms (range } \\
0-12)^{* *}\end{array}$} \\
\hline mean $(95 \% \mathrm{CI})$ & $2.12(1.56-2.67)$ & $2.86(2.25-3.46)$ & 0.079 & $2.41(2.00-2.82)$ & $3.44(2.99-3.88)$ & $<0.001$ \\
\hline
\end{tabular}

* totals may vary because of missing value

**obtained summing the presence of each of 12 possible symptoms reported in the hospital chart

of discomfort and embarrassment due to scars, and changes due to scars, according to types of cancer and gender are shown in Table 4. Among colorectal cancer patients, females reported higher mean scores and percentages, compared to males. Breast cancer patients reported a significantly major discomfort and embarrassment due to their scars and significantly higher percentage of changes in sexual intimacy (44\%) and embarrassment in getting undressed in front of their partner (50\%), than those reported by the other sample. The measure of discomfort and embarrassment appeared strongly associated in all samples ( $r>0.73)$. Among women with breast cancer, mild associations between embarrassment of one's scar and of getting undressed at the gym $(r=0.43)$, and between changes in sexual intimacy and embarrassment in getting undressed in front of partner $(\mathrm{r}=0.38)$ have also been found.

ASI-domains, HADS-subscales and HADS-total, perception of cancer severity and curability, and qol mean T-scores (95\%CI) among patients with breast or colorectal cancer are reported in Table 5. Among colorectal patients, females reported higher mean scores in ASI selfinvestment, ASI appearance stereotyping and HADS anxiety, compared to males. No differences emerged between patients with colorectal cancer or breast cancer, with the exception of perception of cancer severity, perceived less severe for breast cancer patients.
In Table 6, the number and percentage of patients with scores above the theoretical median score (50) in ASI-domains, HADS total, qol and perception of cancer severity and cancer curability are shown according to types of cancer and gender.

The association between psychological distress (HADS-total score $>10)$ and the variables in study has been modelled separately according to types of cancer.

In the multivariate logistic regression model psychological distress was strongly associated with changes - heightened embarrassment when getting undressed in front of one's partner, at the gym, and during sexual intimacy- (OR 19.17; 95\%CI 2.51146.38 ) and frequent resort to self-care behaviours (OR 2.44; 95\%CI 1.29-4.65). High self-investment (ASI-SI $>50$; OR 0.17 ; $95 \%$ CI $0.03-$ 1.00 ) and having had a mastectomy (OR 0.17 ; 95\%CI $0.03-1.03$ ) resulted in low psychological distress among breast cancer patients.

Among colorectal cancer patients psychological distress was associated with a temporary colostomy. No significant association with other clinical or psychological variables were observed. 
Mazzotti E (2019) Embarrassment and behavioural changes among patients living with scars from cancer surgery: The appearance-related side effects and psychological distress

Table 3. Lifestyle and use of non-conventional medicine among patients with breast or colorectal cancer

\begin{tabular}{|c|c|c|c|c|c|c|}
\hline & \multicolumn{2}{|c|}{ Colorectal } & & \multirow{2}{*}{$\begin{array}{c}\text { Colorectal }(\mathrm{N}=71) \\
\text { males+females }\end{array}$} & \multirow{2}{*}{$\begin{array}{c}\text { Breast }(\mathrm{N}=\mathbf{6 4}) \\
\text { females }\end{array}$} & \multirow[b]{3}{*}{ p-value ${ }^{a}$} \\
\hline & males $N=43$ & females $N=\mathbf{2 8}$ & & & & \\
\hline & No.(\%)* & No.(\%)* & p-value & No.(\%)* & No.(\%)* & \\
\hline \multicolumn{7}{|l|}{ Smokers } \\
\hline no & $15(34.88)$ & $15(53.57)$ & & $30(42.25)$ & $36(56.25)$ & \\
\hline yes & $10(23.26)$ & $6(21.43)$ & & $16(22.54)$ & $11(17.19)$ & \\
\hline ex & $18(41.86)$ & $7(25.00)$ & 0.247 & $25(35.21)$ & $17(26.56)$ & 0.267 \\
\hline \multicolumn{7}{|l|}{ Drinkers } \\
\hline no & $31(72.09)$ & $20(71.43)$ & & $51(71.83)$ & $53(82.81)$ & \\
\hline yes & $12(27.91)$ & $8(28.57)$ & 0.951 & $20(28.17)$ & $11(17.19)$ & 0.130 \\
\hline \multicolumn{7}{|l|}{ Vegetables in diet } \\
\hline no & $16(37.21)$ & $4(14.29)$ & & $20(28.17)$ & $7(10.94)$ & \\
\hline yes & 27 (62.79) & $24(85.71)$ & 0.036 & $51(71.83)$ & $57(89.06)$ & 0.012 \\
\hline \multicolumn{7}{|l|}{ Fruits in diet } \\
\hline no & $13(30.23)$ & $8(28.57)$ & & $21(29.58)$ & $10(15.63)$ & \\
\hline yes & $30(69.77)$ & $20(71.43)$ & 0.881 & $50(70.42)$ & $54(84.38)$ & 0.054 \\
\hline \multicolumn{7}{|l|}{ Physical activity } \\
\hline no & $8(18.60)$ & $6(21.43)$ & & $14(19.72)$ & $25(39.06)$ & \\
\hline yes & $35(81.40)$ & $22(8.57)$ & 0.770 & $57(80.28)$ & $39(60.94)$ & 0.013 \\
\hline \multicolumn{7}{|l|}{ Acopuncture } \\
\hline no & $41(95.35)$ & 27 (96.43) & & 68 (95.77) & $61(95.31)$ & \\
\hline yes & $2(.65)$ & $1(3.57)$ & 0.825 & $3(4.23)$ & $3(4.69)$ & 0.896 \\
\hline \multicolumn{7}{|l|}{ Yoga } \\
\hline no & $43(100.0)$ & $28(100)$ & & $71(100.0)$ & 59 (92.19) & \\
\hline yes & $0(0.00)$ & $0(0.0)$ & 1.000 & $0(0.00)$ & $5(7.81)$ & 0.016 \\
\hline \multicolumn{7}{|l|}{ Training autogeno } \\
\hline no & $40(93.02)$ & $28(100)$ & & $68(95.77)$ & $58(90.63)$ & \\
\hline yes & $3(6.98)$ & $0(0.0)$ & 0.153 & $3(4.23)$ & $6(9.38)$ & 0.231 \\
\hline \multicolumn{7}{|l|}{ Massage } \\
\hline no & $41(95.35)$ & $28(100)$ & & $69(97-18)$ & $51(79.69)$ & \\
\hline yes & $2(4.65)$ & $0(0.0)$ & 0.247 & $2(2.82)$ & $13(20.31)$ & 0.001 \\
\hline \multicolumn{7}{|l|}{ Osteopatia } \\
\hline no & $41(95.35)$ & $28(100)$ & & $69(97-18)$ & $60(93.75)$ & \\
\hline yes & $2(4.65)$ & $0(0.0)$ & 0.247 & $2(2.82)$ & $4(6.25)$ & 0.334 \\
\hline \multicolumn{7}{|l|}{ Herbal medicine } \\
\hline no & $35(81.40)$ & $24(85.71)$ & & $59(83.10)$ & $44(68.75)$ & \\
\hline yes & $8(18.60)$ & $4(14.29)$ & 0.635 & $12(16.90)$ & $20(31.25)$ & 0.050 \\
\hline \multicolumn{7}{|l|}{ Psychotherapy } \\
\hline no & $43(100.0)$ & $27(96.43)$ & & $70(98.59)$ & $55(85.94)$ & \\
\hline yes & $0(0.00)$ & $1(3.57)$ & 0.212 & $1(1.41)$ & $9(14.06)$ & 0.005 \\
\hline \multicolumn{7}{|l|}{ Self-care behavior } \\
\hline mean $(95 \% \mathrm{CI})$ & $4.02(3.62-4.23)$ & $4.07(3.59-4.55)$ & 0.877 & $4.04(3.74-4.34)$ & $4.94(4.52-5.36)$ & $<0.001$ \\
\hline \multicolumn{7}{|l|}{ Self-care behavior } \\
\hline no & $24(55.81)$ & $15(53.57)$ & & $39(54.93)$ & $25(39.06)$ & \\
\hline yes & $19(44.19)$ & $13(46.43)$ & 0.853 & $32(45.07)$ & $39(60.94)$ & 0.065 \\
\hline
\end{tabular}

* totals may vary because of missing value

${ }^{a}$ Fisher's exact test 
Mazzotti E (2019) Embarrassment and behavioural changes among patients living with scars from cancer surgery: The appearance-related side effects and psychological distress

Table 4. Descriptive statistics of discomfort and embarrassment of scars according to types of cancer and gender

\begin{tabular}{|c|c|c|c|c|c|c|}
\hline & \multicolumn{2}{|c|}{ Colorectal } & \multirow[b]{2}{*}{ p-value } & \multirow{2}{*}{$\begin{array}{c}\text { Colorectal }(\mathrm{N}=71) \\
\text { males+females }\end{array}$} & \multirow{2}{*}{$\begin{array}{c}\text { Breast }(N=64) \\
\text { females }\end{array}$} & \multirow[b]{2}{*}{ p-value } \\
\hline & males $N=43$ & females $N=28$ & & & & \\
\hline \multicolumn{7}{|l|}{ discomfort about scar } \\
\hline mean $(95 \% \mathrm{CI})$ & $24.42(14.50-34.34)$ & $31.79(17.90-45.67)$ & 0.371 & $27.32(19.36-35.29)$ & $45.40(36.87-54.11)$ & 0.003 \\
\hline \multicolumn{7}{|l|}{ embarassment for scar } \\
\hline mean $(95 \% \mathrm{CI})$ & $20.00(11.35-28.65)$ & $30.71(16.92-44.51)$ & 0.163 & $24.22(16.78-31.67)$ & $44.44(36.09-52.79)$ & $<0.001$ \\
\hline \multicolumn{7}{|l|}{$\begin{array}{l}\text { embarassment to get } \\
\text { undressed (partner) }\end{array}$} \\
\hline no & $29(67.44)$ & $15(53.57)$ & & $44(61.97)$ & $23(35.94)$ & \\
\hline yes & $5(11.63)$ & $6(21.43)$ & & $11(15.49)$ & $32(50.00)$ & \\
\hline n.a. & $9(20.93)$ & $7(25.00)$ & 0.427 & $16(22.54)$ & $9(14.06)$ & $<0.001$ \\
\hline \multicolumn{7}{|l|}{$\begin{array}{l}\text { embarassment to get } \\
\text { undressed (gym) }\end{array}$} \\
\hline no & $16(37.21)$ & $7(25.00)$ & & $23(32.39)$ & $14(21.88)$ & \\
\hline yes & $14(32.56)$ & $14(50.00)$ & & $28(39.44)$ & $39(60.94)$ & \\
\hline n.a. & $13(30.23)$ & $7(25.00)$ & 0.324 & $20(28.17)$ & $11(17.19)$ & 0.044 \\
\hline \multicolumn{7}{|l|}{ changes in intimacy } \\
\hline no & $18(41.86)$ & $5(17.86)$ & & $23(32.39)$ & $27(42.19)$ & \\
\hline yes & $12(27.91)$ & $9(32.14)$ & & $21(29.58)$ & $28(43.75)$ & \\
\hline n.a. & $13(30.23)$ & $14(50.00)$ & 0.088 & $27(38.03)$ & $9(14.06)$ & 0.007 \\
\hline
\end{tabular}

Table 5. Mean $(95 \% \mathrm{CI})$ T-scores of ASI domains, HADS subscales, perception of cancer severity and curability, and qol separately for type of cancer and gender

\begin{tabular}{|c|c|c|c|c|c|c|}
\hline & \multicolumn{2}{|c|}{ Colorectal } & \multirow[b]{2}{*}{ p-value } & \multirow{2}{*}{$\begin{array}{c}\text { Colorectal }(\mathrm{N}=71) \\
\text { males+females }\end{array}$} & \multirow{2}{*}{$\begin{array}{c}\text { Breast }(N=64) \\
\text { females }\end{array}$} & \multirow[b]{2}{*}{ p-value } \\
\hline & males $N=43$ & females $N=\mathbf{2 8}$ & & & & \\
\hline ASI_BIV & $28.02(21.01-35.03)$ & $33.80(25.31-42.28)$ & 0.290 & $30.35(25.04-35.65)$ & $32.31(26.85-37.76)$ & 0.608 \\
\hline ASI_SI & $40.70(32.45-48.94)$ & $51.92(44.09-54.76)$ & 0.067 & $44.93(38.99-50.87)$ & $48.44(42.30-54.58)$ & 0.413 \\
\hline ASI_AS & $30.56(22.66-38.45)$ & $48.15(37.79-58.51)$ & 0.007 & $37.44(30.97-43.90)$ & $38.58(32.14-45.01)$ & 0.805 \\
\hline HADS-anxiety & $30.68(24.30-37.05)$ & $39.46(31-69-47.22)$ & 0.081 & $34.14(29.22-39.05)$ & $39.43(34.96-43.91)$ & 0.117 \\
\hline HADS-depression & $26.80(20.80-32.80)$ & $30.61(25.01-36.22)$ & 0.378 & $28.30(24.12-32.49)$ & $28.20(24.16-32.24)$ & 0.917 \\
\hline HADS-total & $28.74(23.06-34.42)$ & $35.03(28.83-41.23)$ & 0.144 & $31.22(27.04-35.41)$ & $33.82(29.89-37.74)$ & 0.371 \\
\hline qol & $57.36(48.76-65.97)$ & $55.14(44.21-66.08)$ & 0.746 & $56.51(49.91-63.10)$ & $62.01(56.33-67.69)$ & 0.215 \\
\hline severity (R)* & $54.26(47.18-61.35)$ & $53.97(42.20-65.74)$ & 0.963 & $54.15(48.00-60.29)$ & $64.02(57.34-70.70)$ & 0.031 \\
\hline curability & $64.86(58.53-71.19)$ & $58.33(47.74-68.93)$ & 0.256 & $62.28(56.72-67.85)$ & $62.43(55.65-69.22)$ & 0.973 \\
\hline
\end{tabular}

*high score $=$ less severity

$\mathrm{BIV}=$ body image vulnerability; $\mathrm{SI}=$ self-investment; $\mathrm{AS}=$ appearance stereotyping

Table 6. Count and percentage of patients above median score in ASI-domains, HADS, perception of cancer severity and separately for type of cancer and gender

\begin{tabular}{|c|c|c|c|c|c|c|}
\hline & \multicolumn{2}{|c|}{ Colorectal } & \multirow[b]{2}{*}{ p-value } & \multirow{2}{*}{$\begin{array}{c}\text { Colorectal }(\mathrm{N}=71) \\
\text { males+females }\end{array}$} & \multirow{2}{*}{$\begin{array}{c}\text { Breast }(N=64) \\
\text { females }\end{array}$} & \multirow[b]{2}{*}{ p-value } \\
\hline & males $N=43$ & females $N=\mathbf{2 8}$ & & & & \\
\hline \multicolumn{7}{|l|}{ ASI_BIV } \\
\hline$<=50$ & $33(76.74)$ & $21(75.00)$ & & $54(76.06)$ & $48(75.00)$ & \\
\hline$>50$ & $7(16.28)$ & $6(21.43)$ & 0.740 & $13(18.31)$ & $13(20.31)$ & 0.936 \\
\hline \multicolumn{7}{|l|}{ ASI_SI } \\
\hline$<=50$ & $29(67.44)$ & $14(50.00)$ & & $43(60.56)$ & $34(53.13)$ & \\
\hline$>50$ & $14(32.56)$ & $12(42.86)$ & 0.110 & $26(36.62)$ & $27(42.19)$ & 0.634 \\
\hline \multicolumn{7}{|l|}{ ASI_AS } \\
\hline$<=50$ & 34 (79.07) & $15(53.57)$ & & $49(69.01)$ & $48(75.00)$ & \\
\hline$>50$ & $8(18.60)$ & $12(42.86)$ & 0.073 & $20(28.17)$ & $14(21.88)$ & 0.702 \\
\hline \multicolumn{7}{|l|}{ HADS-total } \\
\hline$<=11$ & $16(37.21)$ & $6(21.43)$ & & $22(30.99)$ & $9(14.06)$ & \\
\hline$>11$ & $27(62.79)$ & $22(78.57)$ & 0.160 & $49(69.01)$ & $55(85.94)$ & 0.020 \\
\hline \multicolumn{7}{|l|}{ qol } \\
\hline$<=50$ & $17(39.53)$ & $14(50.00)$ & & $31(43.66)$ & $23(35.94)$ & \\
\hline$>50$ & $26(60.47)$ & $14(50.00)$ & 0.385 & $40(56.34)$ & $41(64.06)$ & 0.360 \\
\hline \multicolumn{7}{|l|}{ severity (R)* } \\
\hline$<=50$ & $22(51.16)$ & $13(46.43)$ & & $35(49.30)$ & $22(34.38)$ & \\
\hline$>50$ & $21(48.84)$ & $15(53.57)$ & 0.697 & $36(50.70)$ & $42(63.63)$ & 0.080 \\
\hline \multicolumn{7}{|l|}{ curability } \\
\hline$<=50$ & $10(23.26)$ & $9(32.14)$ & & $19(26.76)$ & $21(32.81)$ & \\
\hline$>50$ & $33(76.74)$ & $19(67.86)$ & 0.408 & $52(73.24)$ & $43(67.19)$ & 0.442 \\
\hline
\end{tabular}

*high score $=$ less severity

$\mathrm{BIV}=$ body image vulnerability; $\mathrm{SI}=$ self-investment; $\mathrm{AS}=$ appearance stereotyping 
Mazzotti E (2019) Embarrassment and behavioural changes among patients living with scars from cancer surgery: The appearance-related side effects and psychological distress

\section{Conclusions}

Outcomes for patients diagnosed with cancer have improved over the last decades thanks to early detection and innovative therapies. However, most of these patients continued to suffer from the secondary effects of the treatments adopted to cure the cancer. Our results show that appearance-related changes due to breast cancer treatment are associated with high psychological distress. The great attention these women placed on self-care behaviours, such as adopting healthy food in the diet, practicing physical activity, or doing massage and yoga, or psychotherapy, instead of being associated with a reduction was in fact associated with an increase in psychological distress. Moreover, we found a satisfactory psychological status associated with mastectomy, unlike Montazeri [10].

It would appear that breast cancer treatment produced extreme changes in the body image overwhelming every dimension connected with the sense of self. On the one hand, psychological distress appeared to be a relevant issue among our cancer patients, but on the other the results of this study highlight essential differences. To manage psychological distress the first step is to recognize and treat efficiently the disease, although it appears equally important to discover reasons that lead people to a negative psychological status [14]. Our results showed that among breast cancer patients psychological distress is generally tied to changes in sexual relationships and social relationships as well as an excessive investment in self-care behavior, which means that the scar itself becomes secondary; in contrast to our hypothesis, a high investment in one's appearance, meaning self-investment that becomes life-shaping and central to the concept of self and that must be pursued to maximize physical attractiveness, resulted to be associated with psychological well-being.

Among colorectal cancer patients psychological distress is only associated with colostomy. Our study found that the patient's attention and distress is centred on the care of the stoma, unlike Bullen and colleagues [15] who found body image as a predictor of psychopathology in surgical patients with colorectal disease.

The first limitation of the study is the restrained sample size especially of women with colorectal cancer. Future research could explore if psychological distress, embarrassment and change due to scars from cancer surgery is typical of women with breast cancer or it is shared with women with other types of cancer.

In conclusion, a better initial understanding of patients' psychological functioning would help clinicians to develop more tailored therapeutic approaches, and thus would reduce patients' burden in their battle against cancer.

\section{Relevance to clinical practice}

Understanding the difficulties patients with cancer may experience would help clinicians to plan personalized care.

\section{Acknowledgment}

We are very grateful to patients who participated in this study. We are indebted to Prof TF Cash for permission of using the ASI questionnaire, to Sarah Morgan for technical help in reviewing and editing English language, to Cristina Sarandrea for help in recruitment of patients.

\section{Conflicts of interest}

The authors have no conflicts of interest by disclose.

\section{References}

1. Harcourt D, Frith H (2008) Women's experiences of an altered appearance during chemotherapy. Journal of Health Psychology 13: 597-606. [Crossref]

2. Ogden J, Lindridge L (2008) The impact of breast scarring on perception of attractiveness. Journal of Health Psychology 13: 303-310. [Crossref]

3. White CA (2002) Body images in oncology. In: Cash TF, Pruzinsky T, editors. Body image: A handbook of theory, research, \& clinical practice. New York: Guilford Press 379-386.

4. Carver CS, Pozo-Kaderman C, Price AA, Noriega V, Harris SD, et al. (1998) Concern about aspects of body image and adjustment to early stage breast cancer. Psychosomatic Medicine 60: 168-174. [Crossref]

5. Al-Ghazal SK, Fallowfield L, Blamey RW (1999) Does cosmetic outcome from treatment of primary breast cancer influence psychosocial morbidity? European Journal of Surgical Oncology 25: 571-573. [Crossref]

6. Benrud-Larson LM, Heinberg LJ, Boling C, Reed J, White B, et al. (2003) Body image dissatisfaction among women with scleroderma: Extent and relationship to psychosocial function. Health Psychology 22: 130-139. [Crossref]

7. Monaghan SM, Sharpe L, Denton F, Levy J, Schrieber L, et al. (2007) Relationship between appearance and psychological distress in rheumatic diseases. Arthritis and Rheumatism 57: 303-309. [Crossref]

8. Sharpe L, Patel D, Clarke S (2001) The relationship between body image disturbance and distress in colorectal cancer patients with and without stomas. $J$ Psychosom Res 70: 395-402. [Crossref]

9. Mazzotti E, Mozzetta A, V Antinone, S Alfani, G Cianchini, et al. (2011) Psychological distress and investment in one's appearance in patients with pemphigus. J Europ Acad Venereol Dermatol 25: 285-289. [Crossref]

10. Montazeri A (2008) Health-related quality of life in breast cancer patients: bibliographic review of the literature from 1974 to 2007. J Exp Clin Cancer Res 29: 27-32. [Crossref]

11. Zigmond AS, Snaith RP (1983) The Hospital Anxiety and Depression Scale. Acta Psychiatr Scand 67: 361-370. [Crossref]

12. Cash TF, Labarge AS (1996) Development of the appearance schemas inventory: A new cognitive body-image assessment. Cognitive Therapy and Research 20: 37-50.

13. Mazzotti E, Sebastiani C, Marchetti P (2012) Patient-perception of the disease control and relationship with psychological variables. Cancer Management and Research 4: $335-340$

14. Wallace ML, Harcourt D, Rumsey N, Foot A (2007) Managing appearance changes resulting from cancer treatment: Resilience in adolescent females. Psychooncology 16 : 1019-1027. [Crossref]

15. Bullen TL, Sharpe L, Lawsin C, Patel C, Clarke S, et al. (2012) Body image as a predictor of psychopathology in surgical patients with colorectal disease. J Psychosom Res 73: 459-463. [Crossref]

Copyright: (C2019 Mazzotti E. This is an open-access article distributed under the terms of the Creative Commons Attribution License, which permits unrestricted use, distribution, and reproduction in any medium, provided the original author and source are credited. 